Department of Computer Science Technical Report

Botnet Tracking: Exploring a Root-Cause Methodology to Prevent Distributed Denial-of-Service Attacks

Felix C. Freiling and Thorsten Holz and Georg Wicherski 
The publications of the Department of Computer Science of RWTH Aachen University are in general accessible through the World Wide Web.

http://aib.informatik.rwth-aachen.de/ 


\title{
Botnet Tracking: \\ Exploring a Root-Cause Methodology to Prevent Distributed Denial-of-Service Attacks
}

\author{
Felix C. Freiling, Thorsten Holz ${ }^{\star}$ und Georg Wicherski \\ Laboratory for Dependable Distributed Systems, RWTH Aachen University, 52056 Aachen, \\ Germany
}

\begin{abstract}
Zusammenfassung. Denial-of-Service (DoS) attacks pose a significant threat to the Internet today especially if they are distributed, i.e., launched simultaneously at a large number of systems. Reactive techniques that try to detect such an attack and throttle down malicious traffic prevail today but usually require an additional infrastructure to be really effective. In this paper we show that preventive mechanisms can be as effective with much less effort: We present an approach to (distributed) DoS attack prevention that is based on the observation that coordinated automated activity by many hosts needs a mechanism to remotely control them. To prevent such attacks, it is therefore possible to identify, infiltrate and analyze this remote control mechanism and to stop it in an automated fashion. We show that this method can be realized in the Internet by describing how we infiltrated and tracked IRC-based botnets which are the main DoS technology used by attackers today.
\end{abstract}

\section{Introduction}

An important witness of the increasing professionalism in Internet crime are so called Denial-of-Service (DoS) attacks. A DoS attack is an attack on a computer system or network that causes a loss of service to users, typically the loss of network connectivity and services by consuming the bandwidth of the victim network or overloading the computational resources of the victim system [MDDR04]. Using available tools [Dit00], it is relatively easy to mount DoS attacks against remote networks. For the (connection-oriented) Internet protocol TCP, the most common technique is called TCP SYN flooding [SKK ${ }^{+}$97,Com96] and consists of creating a large number of "half open" TCP connections on the target machine, thereby exhausting kernel data structures and making it impossible for the machine to accept new connections. For the (connectionless) protocol UDP, the technique of UDP flooding consists of overrunning the target machine with a large number of UDP packets thereby exhausting its network bandwidth and other computational resources.

Like spam, it is well-known that DoS attacks are extremely hard to prevent because of their "semantic" nature. In the terminology of Schneier [Sch00], semantic attacks target the way we assign meaning to content. For example, it is very hard to distinguish a DoS attack from a peak in the popularity of a large website. Using authentication it is in principle possible to detect and identify the single origin of a DoS attack by looking at the distribution of packets over IP addresses. However, it is almost impossible to detect such an attack if multiple

\footnotetext{
* Thorsten Holz was supported by Deutsche Forschungsgemeinschaft as part of the Graduiertenkolleg "Software for mobile communication systems" at RWTH Aachen University.
} 
attack hosts act in a coordinated fashion against their victim. Such attacks are called Distributed Denial-of-Service (DDoS). DDoS attacks are one of the most dangerous threats in the Internet today since they are not limited to web servers: virtually any service available on the Internet can be the target of such an attack. Higher-level protocols can be used to increase the load even more effectively by using very specific attacks, such as running exhausting search queries on bulletin boards or mounting web spidering attacks, i.e., starting from a given website and then recursively requesting all links on that site.

In the past, there are several examples of severe DDoS attacks. In February 2000, an attacker targeted major e-commerce companies and news-sites [Gar00]. The network traffic flooded the available Internet connection so that no users could access these websites for several hours. In recent years, the threat posed by DDoS attacks grew and began to turn into real cybercrime. An example of this professionalism are blackmail attempts against a betting company during the European soccer championship in 2004 [BBC04]. The attacker threatened to take the website of this company offline unless the company payed money. Similar documented cybercrime cases happened during other major sport events. Furthermore, paid DDoS attacks to take competitor's websites down were reported in 2004 [FBI04]. These type of attacks often involve so called botnets [McC03], i.e., networks of compromised machines that are remotely controlled by an attacker. Botnets often consist of several thousand machines and enable an attacker to cause serious damage. Botnets are regularly used for DDoS attacks since their combined bandwidth overwhelms the available bandwidth of most target systems. In addition, several thousand compromised machines can generate so many packets per second that the target is unable to respond to so many requests.

Defensive measures against DDoS can be classified as either preventive or reactive [MR04]. Currently, reactive techniques dominate the arena of DDoS defense methods (the work by Mirkovic et al. [MDDR04] gives an excellent survey over academic and commercial systems). The idea of reactive approaches is to detect the attack by using some form of (distributed) anomaly detection on the network traffic and then react to the attack by reducing the malicious network flows to manageable levels [MRRK03]. The drawback of these approaches is that they need an increasingly complex and powerful sensing and analysis infrastructure to be effective: the approach is best if large portions of network traffic can be observed for analysis, preferably in real-time.

Preventive methods either eliminate the possibility of a DDoS attack altogether or they help victims to survive an attack better by increasing the resources of the victim in relation to those of the attacker, e.g., by introducing some form of strong authentication before any network interaction can take place (see for example work by Meadows [Mea98]). Although being effective in theory, these survival methods always boil down to an arms race between attacker and victim where the party with more resources wins. In practice, it seems as if the arms race is always won by the attacker, since it is usually easier for him to increase his resources (by compromising more machines) than for the victim, which needs to invest money in equipment and network bandwidth.

Preventive techniques that aim at DDoS attack avoidance (i.e., ensuring that DDoS attacks are stopped before they are even launched) have received close to 
no attention so far. One reason for this might be the popular folklore that the only effective prevention technique for DDoS means to fix all vulnerabilities in all Internet hosts that can be misused for an attack (see for example Section 5 of [MR04]). In this paper we show that this folklore is wrong by presenting an effective approach to DDoS prevention that neither implies a resource arms race nor needs any additional (authentication) infrastructure. The approach is based on the observation that coordinated automated activity by many hosts is at the core of DDoS attacks. Hence the attacker needs a mechanism to remotely control a large number of machines. To prevent DDoS attacks, our approach attempts to identify, infiltrate and analyze this remote control mechanism and to stop it in an automated and controlled fashion. Since we attack the problem of DDoS at the root of its emergence, we consider our approach to be a root-cause method to DDoS defense.

It may seem unlikely that it is possible to automatically analyze and infiltrate a malicious remote control method crafted by attackers for evil purposes. However, we provide evidence of the feasibility of our strategy by describing how we successfully tracked and investigated the automated attack activity of botnets in the Internet. The idea of our methods is to "catch" malware using honeypots, i.e., network resources (computers, routers, switches, etc.) deployed to be probed, attacked, and compromised. Honeypots run special software which permanently collects data about the system behavior and facilitates automated post-incident forensic analysis. From the automated analysis we derive the important information necessary to observe and combat malicious actions of the botnet maintainers. In a sense, our approach can be characterized as turning the methods of the attackers against themselves.

The paper is structured as follows: Section 2 gives a brief overview over botnets and their usage for DDoS attacks. In Section 3 we introduce a general methodology to prevent DDoS attacks and exemplify a technical realization in Section 4. We present our results in Section 5 and conclude this paper with Section 6 .

\section{Distributed Denial-of-Service using Botnets}

In this section we give a brief overview over botnets and how they can be used to mount DDoS attacks. More technical details can be found in [The05]. A botnet is a network of compromised machines running programs (usually referred to as bot, zombie, or drone) under a common Command and Control (C\&C) infrastructure. Usually, the controller of the botnet compromises a series of systems using various tools and then installs a bot to enable remote control of the victim computer via Internet Relay Chat (IRC).

Newer bots can even automatically scan whole network ranges and propagate themselves using vulnerabilities and weak passwords on other machines. After successful invasion, a bot uses Trivial File Transfer Protocol (TFTP), File Transfer Protocol (FTP), HyperText Transfer Protocol (HTTP), or CSend (an IRC extension to send files to other users) to transfer itself to the compromised host. The binary is started and tries to connect to the hard-coded master IRC server on a predefined port, often using a server password to protect the botnet infrastructure. This server acts as the $\mathrm{C} \& \mathrm{C}$ server to manage the botnet. Often 


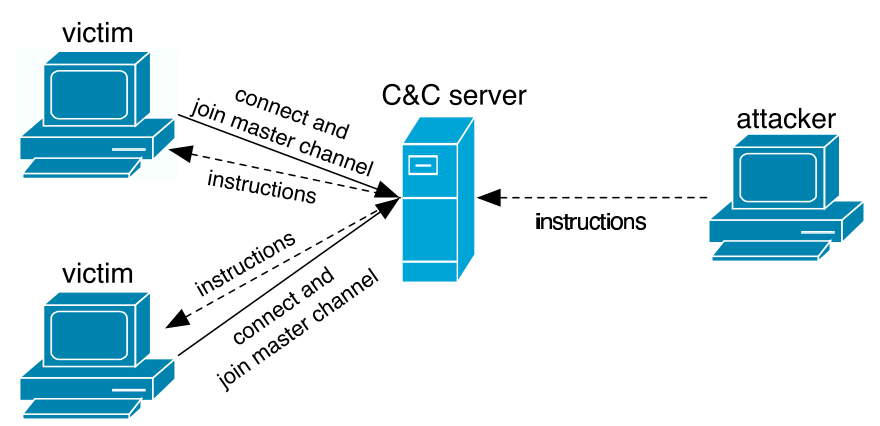

Abb. 1. Communication flow in a botnet

a dynamic DNS name is provided rather than a hard coded IP address, so the bot can be easily relocated. Using a specially crafted nickname, the bot tries to join the master's channel, often using a channel password, too. In this channel, the bot can be remotely controlled by the attacker.

Commands can be sent to the bot in two different ways: via sending an ordinary command directly to the bot or via setting a special topic in the channel that all bots interpret. For example, the topic

advscan lsass 20050 -b

tells the bots to spread further with the help of a known vulnerability (the Windows lsass vulnerability). The bots should start 200 concurrent threads that should scan with a delay of 5 seconds for an unlimited time (parameter 0 ). The scans should target machines within the same Class B network (parameter $-b$ ). As another example, the topic

http.update http://<server>/rBot.exe c:\msy32awds.exe 1

instructs the bots to download a binary from the Internet via HTTP to the local filesystem and execute it (parameter 1).

If the topic does not contain any instructions for the bot, then it does nothing but idling in the channel, awaiting commands. That is fundamental for most current bots: they do not spread if they are not told to spread in their master's channel. Figure 1 depicts the typical communication flow in a botnet.

In order to remotely control the bots, the controller of a botnet has to authenticate himself before issuing commands. This authentication is done with the help of a classical authentication scheme. At first, the controller has to login with his username. Afterwards, he has to authenticate with the correct password to approve his authenticity. The whole authentication process is only allowed from a predefined domain, so that only certain people can start this process. Once an attacker is authenticated, he has complete control over the bots and can execute arbitrary commands.

Today, botnets are most often used to mount DDoS attacks in the Internet. All common bots include several different possibilities to participate in these attacks. Most commonly implemented, and also very often used, are TCP SYN $\left[\mathrm{SKK}^{+} 97, \mathrm{Com} 96\right]$ and UDP flooding attacks. For example, the command

ddos.syn XXX.XXX.XXX.XXX 80600 
instructs the bots within the botnet to start a TCP SYN flooding attack against the specified IP address against TCP port 80 for 600 seconds. Another example is the following command:

udp XXX.XXX.XXX.XXX 1800050000100

It instructs the bots to mount a UDP flooding attack against the specified target with 18,000 packets of a size of 50,000 bytes using a delay of 100 milliseconds between each packet. Note that the C\&C IRC server that is used to connect all bots is in most cases also a compromised machine.

\section{Preventing Distributed Denial-of-Service Attacks}

In this section we introduce a general methodology to prevent DDoS attacks. It is based on the following line of reasoning:

1. To mount a successful DDoS attack, a large number of compromised machines are necessary.

2. To coordinate a large number of machines, the attacker needs a remote control mechanism.

3. If the remote control mechanism is disabled, the DoS attack is prevented.

We will substantiate this line of reasoning in the following paragraphs.

\subsection{A Large Number of Machines is Necessary}

Why does an attacker need a large number of machines to mount a successful DDoS attack? If an attacker controls only few machines, a DDoS attack is successful only if the total resources of the attacker (e.g., available bandwidth or possibility to generate many packets per second) are greater than the resources of the victim. Otherwise the victim is able to cope with the attack. Hence, if this requirement is met, the attacker can efficiently overwhelm the services offered by the victim or cause the loss of network connectivity.

Moreover, if only a small number of attacking machines are involved in an attack, these machines can be identified and counteractive measures can be applied, e.g., shutting down the attacking machines or blocking their traffic. To obfuscate the real address of the attacking machines, IP spoofing, i.e., sending IP packets with a counterfeited sender address, is often used. Furthermore, this technique is used to disguise the actual number of attacking machines by seemingly increasing it. However, IP spoofing does not help an attacker to conduct a DDoS attack from an efficiency point of view. It does not increase the available resources, but it even reduces them due to computing efforts for counterfeiting the IP addresses. In addition, several ways to detect and counteract spoofed sender address exist, e.g., ingress filtering [Fer00], packet marking [SP01], or ICMP traceback [Bel01,SWKA00]. The IP distribution of a large number of machines in different networks makes ingress filter construction, maintenance, and deployment much more difficult. Additionally, incident response is hampered by a high number of separate organizations involved.

So control over a large number of machines is necessary for a successful DDoS attack. 


\subsection{A Remote Control Mechanism is Necessary}

The success of a DDoS attack depends on the volume of the malicious traffic as well as the time this traffic is directed against the victim. Therefore, it is vital that the actions of the many hosts which participate in the attack are wellcoordinated regarding the type of traffic, the victim's identity, as well as the time of attack.

A cautious attacker may encode all this information directly into the malware which is used to compromise the zombies that form the DDoS network. While this makes him harder to track down, the attacker looses a lot of flexibility since he needs to plan his deeds well in advance. Additionally, this approach makes the DDoS attack also less effective since it is possible to analyze the malware and then reliably predict when and where an attack will take place. Therefore it is desirable to have a channel through which this information can be transferred to the zombies on demand, i.e., a remote control mechanism.

A remote control mechanism has many more advantages:

1. The most effective attacks come by surprise regarding the time, the type and the target of attack. A remote control mechanism allows an attacker to react swiftly to a given situation, e.g., to mount a counterattack or to substantiate blackmail threats.

2. Like any software, malware is usually far from perfect. A remote control mechanism can be used as an automated update facility, e.g., to upgrade malware with new functionality.

In short, a DDoS attack mechanism is only effective if an attacker has some type of remote control over a large number of machines. Then he can issue commands to exhaust the victim's resources at many systems, thus successfully attacking the victim.

\subsection{Preventing Attacks}

Our methodology to mitigate DDoS attacks aims at manipulating the root-cause of the attacks, i.e., influencing the remote control network. Our approach is based on three steps:

1. Infiltrating the remote control network.

2. Analyzing the network in detail.

3. Shutting down the remote control network.

In the first step, we have to find a way to smuggle an agent into the control network. In this context, the term agent describes a general procedure to mask as a valid member of the control network. This agent must thus be customized to the type of network we want to plant it in. The level of adaptation to a real member of the network depends on the target we want to infiltrate. For instance, to infiltrate a botnet we would try to simulate a valid bot, maybe even emulating some bot commands.

Once we are able to sneak an agent into the remote control network, it enables us to perform the second step, i.e., to observe the network in detail. So we can start to monitor all activity and analyze all information we have collected. 
In the last step, we use the collected information to shut down the remote control network. Once this is done, we have deprived the attacker's control over the other machines and thus efficiently stopped the threat of a DDoS attack with this network. Again, the particular way in which the network is shut down depends on the type of network.

\subsection{Discussion}

The methodology described above can be applied to different kinds of remote control networks and is thus very general. The practical challenge of the methodology is to automate the infiltration and analysis process as much as possible. In all these cases, the zombies need to establish a communication channel between themselves and the attacker. If it is possible to "catch" this malware in a controlled way, it is possible to extract a lot of information out of it in an automated fashion. For example, if contact to the attacker is set up by establishing a regular network connection, the network address of the attacker's computer can be automatically collected.

To many readers, the methodology may sound like coming directly from a James Bond novel and it is legitimate to ask for evidence of its feasibility. In the following section we give exactly this evidence. We show that this method can be realized in the Internet by describing how we infiltrated and tracked IRC-based botnets which are the main DDoS technology used by attackers today.

\section{An Example: Tracking Botnets}

In this section we exemplify a technical realization of the methodology we introduced above. We present an approach to track and observe botnets that is able to prevent DDoS attacks.

As already stated in the last section, tracking botnets is clearly a multi-step operation: First one needs to gather some data about an existing botnet. This can for instance be obtained with the help of botnets or via an analysis of captured malware. With the help of this information it is possible to smuggle a client into the network.

We first introduce two techniques to retrieve the necessary information from a botnet which enables us to infiltrate in it. The necessary information includes:

- DNS/IP-address of IRC server and port number.

- Password to connect to IRC-server (optional).

- Nickname of a bot and ident [Joh93] structure.

- Name of IRC channel to join and (optional) channel password.

The first method to retrieve this information is based on honeypot technology and is presented in Section 4.1. The second method is more lightweight and presented in Section 4.2. Then we describe the observation and analysis process in which we collected further information (Section 4.3). Finally, in Section 4.4 we give a small overview of possible ways to shut down a botnet. 


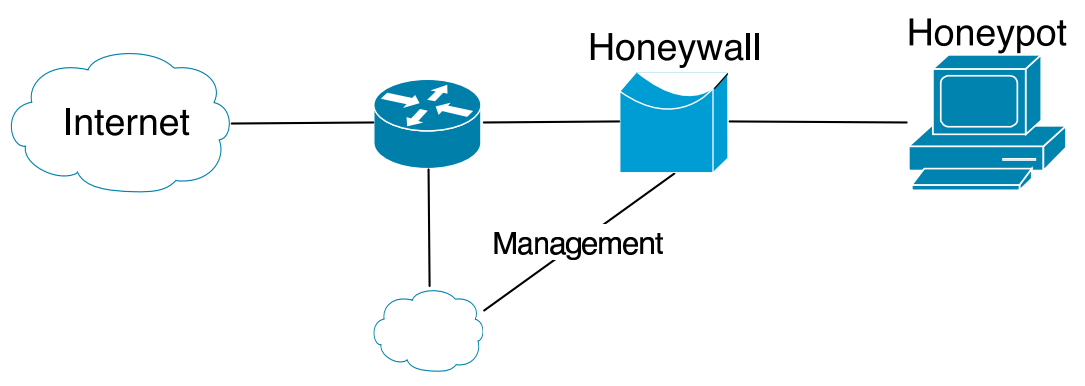

Abb. 2. Setup for tracking botnets

\subsection{Collecting Malware with Honeypots}

A honeypot is a network resource (computers, routers, switches, etc.) deployed to be probed, attacked, and compromised. A honeynet is a network of honeypots. Honeypots run special software which permanently collects data about the system behavior and facilitates automatic post-incident forensic analysis. The collected data enables us to determine the necessary information about an existing botnet. A detailed introduction to honeypots can for example be found in [DGH04].

Using a so called GenII Honeynet [The03] containing some Windows honeypots, we are able to collect all necessary information. We deployed a typical GenII Honeynet with some small modifications as depicted in Figure 4.1.

The Windows honeypot runs an unpatched version of Windows 2000 or Windows XP. This system is thus very vulnerable to attacks. It is located within the internal network of RWTH Aachen University. On average, the expected lifespan of the honeypot is less than ten minutes. After this small amount of time, the honeypot is often successfully exploited by automated malware. The shortest compromise time was only a few seconds: Once we plugged the network cable in, a bot compromised the machine and installed itself on the machine.

As explained in the previous section, a bot tries to connect to the $\mathrm{C} \& \mathrm{C}$ server to obtain further commands once it successfully attacked the honeypot. This is where the Honeywall comes into play. The Honeywall is a transparent bridge that enables the two tasks Data Control and Data Capture. Due to the Data Control facilities, it is possible to control the outgoing traffic. Using available tools for Data Control we can replace all suspicious in- and outgoing messages. A message is suspicious if it contains typical IRC messages for command and control, for example "TOPIC", "PRIVMSG", or " NOTICE". Thus we are able to inhibit the bot from accepting valid commands from the master channel. It can therefore cause no harm to others and therefore we have caught a bot inside our Honeynet. As a side effect, we can also derive all necessary sensitive information for a botnet from the data we have obtained up to that point in time: The Data Capture capability of the Honeywall allows us to determine the DNS/IPaddress the bot wants to connect to and also the corresponding port number. In addition, we can derive from the Data Capture logs the nickname, the ident information, the server's password, channel name, and the channel password as well. So we have collected all necessary information and the honeypot can catch further malware. Since we do not care about the captured malware for now, we rebuild the honeypot every 24 hours to have a "clean" system every day. This 
has proven to be a good time span since after this amount of time the honeypot tends to become unstable due to installed malware.

\subsection{Collecting Malware with mwcollect}

The approach described in the previous section works, but has several drawbacks:

- A honeypot will crash regularly if the bot fails to exploit the offered service, e.g. due to a wrong offset within the exploit.

- The honeypot itself has to be closely monitored in order to detect changes on the system. Furthermore, these changes have to be analyzed carefully to detect malware.

- The approach does not scale well; observing a large number of IP addresses is difficult.

To overcome these limitations, we developed a program called mwcollect to capture malware in non-native environments. This tool simulates several vulnerable services and waits for them to be exploited. It is comparable to a lowinteraction honeypot like honeyd [Pro04]. In contrast to honeyd it is tailored to collecting of malware and offers possibilities that honeyd cannot offer, e.g. better packet handling and more flexibility.

mwcollect is based upon a very flexible and modularized design. The core module - the actual daemon - handles the network interface and coordinates the actions of the other modules. Furthermore, the core module implements a sniffer mode which records all traffic to a special log file. This can for example be useful if an unknown exploit is detected that needs to be further analyzed.

Several modules, which register themselves in the core, fulfill the actual tasks. There are basically four types of modules:

- Vulnerability modules open some common vulnerable ports (e.g. TCP Port 135 or 2745) and simulate the vulnerabilities according to these ports.

- Shellcode parsing modules analyze the shellcode, an assembly language program which executes a shell, received by one of the vulnerability modules. These modules try to extract generic URLs from the shellcode.

- Fetch modules simply download the files specified by an URL. These URLs do not necessarily have to be HTTP or FTP URLs, but can also be TFTP or other protocols.

- Submission modules handle successfully downloaded files, for example by writing it to disk or submitting it to a database.

Vulnerability modules seem to be the most important part of mwcollect, but in fact they are not more important than every other module, they all require each other. Moreover, the vulnerable service emulation is not very sophisticated, but functional: Often malware does not require an indistinguishable emulation of a real service but an approximation of it. In most cases it is thus sufficient to provide some minimal information at certain offsets in the network flow. This information is used by the malware to calculate the offsets it can use to exploit the service. Upon successful exploitation, the payload of the malware is passed to another kind of modules. 
Currently there is only one shellcode parsing module that is capable of analyzing all shellcodes we have found up to now. The module first recursively detects $X O R$ decoders in a generic way. An XOR decoder is a common way to encrypt the actual shellcode in order to evade intrusion detection systems. Afterwards the module decodes the code itself according to the computed key and then applies some pattern detection, for example CreateProcess and URLDownloadToFileA detection patterns. The results are further analyzed and if an URL is detected, it is passed to the fetch modules. A module that parses shellcodes in an even more generic way by emulating a Windows Operating System environment is currently under development.

Fetch modules have the simple task of downloading files from the Internet. There are currently three different fetch modules: one for TFTP, one for generic HTTP and FTP URLs and finally one for CSend and similar transfer methods used by different species of bots.

Finally, submission modules handle successfully downloaded files. Currently there are three different types of submission modules:

- A module that stores the file in a configurable location on the filesystem and is also capable of changing the ownership.

- A module that submits the file to a central database to enable distributed sensors with central logging interface

- A module that checks the file with the help of different anti-virus scanners for known malware. Optionally this module sends an alert to enable an early warning system. Therefore, mwcollect can also be seen as a kind of intrusion detection system.

Two further features of mwcollect are important to efficiently collect malware: virtualized filesystem and shell emulation.

A common technique to infect a host via a shell is to write commands for downloading and executing malware into a temporary file and then execute this file. Therefore a virtual filesystem was implemented to enable this type of attacks. Every shell session has its own virtual filesystem so concurrent infection sessions using similar exploits do not conflict. The temporary file is analyzed and the malware is downloaded from the Internet in an automated way.

Some Malware does not spread by download shellcodes but by providing a shell to the attacker. Therefore it is sometimes required to spawn and emulate a Windows shell. Shell emulation is centralized in the core module since only one type of shell is emulated. However, modules can register additional commands that extend the possibilities for the malware. mwcollect currently simulates a rudimentary shell and implements several commands: echo, ftp.exe and tftp.exe, as well as batch file execution.

The big advantage of using mwcollect to collect malware is clearly both stability and scalability: A bot trying to exploit a honeypot running Windows 2000 with payload that targets Windows XP will presumably crash the service. In most cases, the honeypot will be forced to reboot. In contrast to this, mwcollect can be successfully exploited by all of those tools and hence catch a lot more binaries this way. Furthermore, mwcollect can listen on many IP addresses in parallel. We tested the program with 256 IP addresses and it scaled well. 
To derive the sensitive information of the botnet from the collected malware, a further analysis is necessary. A possible way to extract the information from the captured malware is reverse engineering, the process of carefully analyzing a program without having its source code. This process is time consuming, but we have developed some techniques that enables us to extract the information within a few minutes. A better approach is an automated analysis with the help of a honeynet. The setup depicted in Figure 4.1 can be used for this purpose. Upon startup, the Windows honeypot downloads a piece of malware from a database located somewhere in the Internet. It executes the file and reboots itself after a few minutes. During this time span, the bot installs itself on the honeypots and connects to the $\mathrm{C} \& \mathrm{C}$ server. With the help of the Honeywall, we are again able to extract all necessary information. In addition, the honeypot resets the hard disk during each reboot so that a clean image is booted each time.

In a third approach, we are currently implementing a virtual machine that implements an environments in which the bot can be executed. This virtualization emulates a Windows environment and enables us to efficiently analyze the malware.

\subsection{Observing Botnets}

Once we have collected all sensitive information of the botnet, we start to infiltrate the botnet as we have all the necessary data. In a first approach, it is possible to setup a normal IRC client and try to connect to the network. If the operators of the botnets do not detect this client, logging of all commands can be enabled. This way, all bot commands and all actions can be observed. If the network is relatively small (i.e., less then 50 clients), there is a chance that the bogus client will be identified since it does not answer to valid commands. In this case, the operators of the botnets tend to either ban and/or DDoS the suspicious client.

But there are many problems with this approach: Some botnets use very strongly stripped down $\mathrm{C} \& \mathrm{C}$ server which is not RFC compliant so that a normal IRC client can not connect to this network. A possible way to circumvent this situation is to find out what the operator has stripped out, and modify the source code of the IRC client to override it. Furthermore, this approach does not scale very well. Tracking more than just a few botnets is not possible since a normal IRC client will be overwhelmed with the amount of logging data and it does not offer a concise overview of what is happening.

Therefore we use an IRC client optimized for botnet tracking called drone. This software was developed by two members of the German Honeynet Project and offers several decent techniques for observing botnets:

- Multi-server support to track a large number of botnets in parallel

- Excessive debug-logging interface so that it is possible to get information about RFC non-compliance issues very fast and fix them in the client

- Automated downloading of malware identified within the botnet

- Modular interface to un/load modules at runtime

Furthermore, drone is capable of using SOCKS v4 proxies so we do not run into problems if it's presence is noticed by an attacker in a botnet. The SOCKS 
$\mathrm{v} 4$ proxies are on dial-in accounts in different networks so that we can easily change the IP addresses of our infiltrated bot.

When observing more than a couple of networks, we began to check if some of them are linked, and group them if possible. Link-checking is simply realizable: our client just joins a specific channel on all networks and detects if more than one client is there, thus concluding the the networks controlled by several C\&C servers are linked. Surprisingly, many networks are linked.

\subsection{Preventing DDoS attacks caused by Botnets}

Several ways to prevent DDoS attacks caused by botnets exist that we want to sketch in this section. Since we observe the communication flow within the botnet, we are also able to observe the IP addresses of the bots unless this information is obfuscated, e.g., by modifying the $\mathrm{C} \& \mathrm{C}$ server. Thus one possible way to stop DDoS attacks with this methodology is to contact the owner of the compromised system. This is however a tedious and cumbersome job, since many organizations are involved and these organizations are spread all over the world. In addition, the large number of bots make this approach nearly infeasible, only an automated notification system could help.

Another approach to prevent DDoS attacks caused by botnets aims at stopping the actual infrastructure, in particular the $\mathrm{C} \& \mathrm{C}$ server, since this component is vital for the remote control network. One possible way to stop the $\mathrm{C} \& \mathrm{C}$ server is described in [Fis05]: Most botnets use a dynamic DNS name instead of a hardcoded IP address for the C\&C server. So if the DNS name is changed so that it resolves to an IP address in a private subnet as defined in RFC 1918, the bots are not able to connect to the central server. Thus the remote control network is efficiently shut down. For this approach, the assistance of the DNS provider is needed, though.

In addition, the collected information about botnets enable another way to stop the botnet. We know the IP address of the C\&C server and are thus able to locate it. If the operator of the network cooperates, it is possible to shut down this server and thus shutting down the remote control network.

\section{Results}

In this section we present some of the findings we obtained through our observation of botnets. Data is sanitized so that it does not allow one to draw any conclusions about specific attacks against a particular system, and protects the identity and privacy of those involved. The information about specific attacks and compromised systems was forwarded to DFN-CERT (Computer Emergency Response Team) based in Hamburg, Germany.

The results are based on the observations collected with just two sensors. One sensors uses the approach depicted in Section 4.1 and is located within the network of RWTH Aachen University. The other sensor is based on the technique and software introduced in Section 4.2 and is located within a dial-in network of a German ISP.

We start with some statistics about the botnets we have observed in the last five months: 
- Number of botnets: We were able to track about 180 botnets during the last five months. Some of them went offline (e.g. C\&C server went offline or inexperienced attackers) and at the time of writing (March 2005) we are tracking about 60 active botnets.

- Number of hosts: During these few months, we saw more than 300,000 unique IP addresses joining at least one of the channels we monitored. Seeing an IP means here that the $\mathrm{C} \& \mathrm{C}$ server was not modified to not send a JOIN message for each joining client. If an IRC server is modified not to show joining clients in a channel, we do not see IPs here. Furthermore some IRC server obfuscate the joining clients IP address and obfuscated IP addresses do not count as seen, too. This shows that the threat posed by botnets is probably worse than originally believed. Even if we are very optimistic and estimate that we track a significant percentage of all botnets and all of our tracked botnet C\&C servers are not modified to hide JOINs or obfuscate the joining clients IPs, this would mean that more than one million hosts are compromised and can be controlled by malicious attackers.

- Typical size of Botnets: Some botnets consist of only a few hundred bots. In contrast to this, we have also monitored several large botnets with up to 50,000 hosts. The actual size of such a large botnet is hard to estimate. Often the attackers use heavily modified IRC servers and the bots are spread across several $\mathrm{C} \& \mathrm{C}$ servers which are linked together to form a common remote control network. We use link-checking between IRC servers to detect connections between different botnets that form one large botnet. Thus we are able to approximate the actual size.

As a side note: We know about a home computer which got infected by 16 different bots, so its hard to make an estimation about world bot population here.

- Dimension of DDoS-attacks: We are able to make an educated guess about the current dimension of DDoS-attacks caused by botnets. We can observe the commands issued by the controllers and thus see whenever the botnet is used for such attacks. From the beginning of November 2004 until the end of March 2005, we were able to observe 406 DDoS-attacks against 179 unique targets. Often these attacks targeted dial-up lines, but there are also attacks against bigger websites or other IRC server.

- Spreading of botnets: Commands issued for further spreading of the bots are the most frequent observed messages. Commonly, Windows systems are exploited and thus we see most traffic on typical Windows ports used for file sharing.

- "Updates" within botnets: We also observed updates of botnets quite frequently. Updating in this context means that the bots are instructed to download a piece of software from the Internet and then execute it.

We conclude that our general methodology described in Section 3 is feasible and the automated approach described in Section 4.2 is effective. We collected more than 5500 binaries (about 800 unique ones) with mwcollect in just one week on a single sensor. This sensor has only one IP address and is connected to the Internet via a German DSL dial-in provider with 4 MBit downstream and 2 MBit upstream. About five percent of the unique files were broken due to failures during TFTP transfer. We are currently in the process of analyzing 
these files. Once we have implemented a virtualization mechanism to efficiently and automatically analyze the collected files, we hope to be able to significantly increase the number of botnets we observe. In addition, this information can be used to prevent DDoS attacks by shutting down the C\&C server.

\section{Conclusion and Further Work}

DDoS attacks have become increasingly dangerous in recent years and we are observing a growing professionalism in the type of Internet crime surrounding DDoS. In this paper we have introduced a technique for DDoS attack prevention that neither implies a resource arms race nor needs any additional infrastructure. In contrast to previous work in this area our approach is preventive instead of reactive. Our technique attacks a root-cause of DDoS attacks: in order to be effective, an attacker has to control a large number of machines and thus needs a remote control network. Our methodology aims at shutting down this control network by infiltrating it and analyzing it in detail.

We have exemplified a technical realization of this methodology considering as example the tracking of IRC-based botnets. Such a botnet is a network of compromised machines that can be remotely controlled by an attacker through Internet relay chat technology. Due to their immense size (tens of thousands of systems can be linked together), these botnets pose a severe threat to the Internet community, e.g., since their aggregated resources can be used to overwhelm most targets with a DDoS attack. We have shown that an automation of this approach is possible to a high degree. With the help of honeypots, i.e., network resources deployed to be compromised, we are able to automate the process of collecting sensitive information of the remote control network by automatically "collecting" malware. Via an automated analysis of the captured binaries we are furthermore able to extract the sensitive information that allow to shut down the control network.

With the help of just two sensors we were able to track a significant number of botnets within a few months. In the future we want to analyze how good our approach scales. Therefore we want to deploy more sensors within different networks. In addition, we aim at speeding up the automated analysis process so that it becomes even more effective. This can for example be achieved with the help of a generic shellcode parser or a virtual machine that analyzes and extracts the sensitive information from the captured binaries.

Moreover, the data we captured while observing the botnets show that these control networks are used for more than just DDoS attacks. Possible usages of botnets can be categorized as listed below. And since a botnet is nothing more then a tool, there are most likely other potential uses that we have not listed:

- Spamming: Some bots offer the possibility to open a SOCKS v4/v5 proxy a generic proxy protocol for TCP/IP-based networking applications - on a compromised machine. After having enabled the SOCKS proxy, this machine can then be used for nefarious tasks such as sending bulk email (spam) or phishing mails. With the help of a botnet and thousands of bots, an attacker is able to send massive amounts of spam. Some bots also implement a special function to harvest email-addresses from the victims. 
- Attacking IRC Chat Networks: Botnets are also used for DDoS attacks against Internet Relay Chat (IRC) networks. Popular among attackers is especially the so called clone attack: In this kind of attack, the controller orders each bot to connect a large number of clones to the victim IRC network. The victim is overwhelmed by service request from thousands of (cloned) bots.

- Manipulating online polls/games: Online polls/games are getting more and more attention and it is rather easy to manipulate them with botnets. Since every bot has a distinct IP address, every vote will have the same credibility as a vote cast by a real person. Online games can be manipulated in a similar way.

- Sniffing Traffic: Bots can also use a packet sniffer to watch for interesting clear-text data passing by a compromised machine. The sniffers are mostly used to retrieve sensitive information like usernames and passwords.

- Keylogging: If the compromised machine uses encrypted communication channels (e.g. HTTPS or POP3S), then just sniffing the network packets on the victim's computer is useless since the appropriate key to decrypt the packets is missing. But most bots also implement functions to log keystrokes. With the help of a keylogger it is very easy for an attacker to retrieve sensitive information.

- Harvesting of information: Sometimes we can also observe the harvesting of information from all compromised machines. With the help of special commands the operator of the botnet is able to request a list of sensitive information from all bots.

With our method we can shut down the root-cause of all of these types of nuisances, and hence our method is not restricted to combat DDoS.

In the future, we hope to develop more advanced honeypots that help us to gather more information about threats such as botnets. Examples include clientside honeypots that actively participate in networks (e.g. by crawling the web, idling in IRC channels, or using P2P-networks) or modify honeypots so that they capture malware and send it to anti-virus vendors for further analysis. It is also to be expected that future botnets will use communication facilities other than IRC (like potentially decentralized p2p-communication). Our methodology seems valid also for these scenarios, although more research in this area is still needed.

\section{Acknowledgments}

We thank Lucia Draque Penso for reading a previous version of this paper and giving valuable feedback that substantially improved it's presentation. In addition, we would like to thank the authors of drone, an IRC client optimized for botnet tracking, for their hard word in writing the program.

\section{Literatur}

[BBC04] Hacker threats to bookies probed. Internet: http://news.bbc.co.uk/1/hi/ technology/3513849.stm, Accessed March 2005, February 2004.

[Bel01] Steve M. Bellovin. ICMP traceback messages, March 2001. Internet Draft.

[Com96] Computer Emergency Response Team. CERT advisory CA-1996-21 TCP SYN Flooding Attacks. Internet: http://www.cert.org/advisories/CA-1996-21.html, 1996. 
[DGH04] Maximillian Dornseif, Felix C. Gärtner, and Thorsten Holz. Vulnerability assessment using honepots. Praxis der Informationsverarbeitung und Kommunikation (PIK), 4(27):195-201, 2004.

[Dit00] Dave Dittrich. Distributed Denial of Service (DDoS) attacks/tools resource page. Internet: http://staff .washington.edu/dittrich/misc/ddos/, 2000.

[FBI04] FBI report on Operation Cyberslam. Internet: http://www.reverse.net/ operationcyberslam.pdf, Accessed March 2005, February 2004.

[Fer00] Paul Ferguson. Network ingress filtering: Defeating denial of service attacks which employ ip source address spoofing, May 2000. Request for Comments: RFC 2827.

[Fis05] Tom Fischer. Botnetze. In Proceedings of 12th DFN-CERT Workshop, March 2005.

[Gar00] Lee Garber. Denial-of-service attacks rip the internet. Computer, 33(4):12-17, April 2000 .

[Joh93] M. St. Johns. Identification protocol, February 1993. Request for Comments: RFC 1413.

[McC03] Bill McCarty. Botnets: Big and bigger. IEEE Security E Privacy, 1(4):87-90, 2003.

[MDDR04] Jelena Mirkovic, Sven Dietrich, David Dittrich, and Peter Reiher. Internet Denial of Service: Attack and Defense Mechanisms. Prentice Hall PTR, 2004.

[Mea98] Catherine Meadows. A formal framework and evaluation method for network denial of service. In Proceedings of the 1999 IEEE Computer Security Foundations Workshop, pages 4-13. IEEE Computer Society Press, 1998.

[MR04] Jelena Mirkovic and Peter Reiher. A taxonomy of DDoS attacks and defense mechanisms. ACM SIGCOMM Computer Communications Review, 34(2):39-54, April 2004.

[MRRK03] J. Mirkovic, M. Robinson, P. Reiher, and G. Kuenning. Alliance formation for DDoS defense. In Proceedings of the New Security Paradigms Workshop 2003. ACM SIGSAC, August 2003.

[Pro04] Niels Provos. A virtual honeypot framework. In Proceedings of 13th USENIX Security Symposium, 2004.

[Sch00] Bruce Schneier. Inside risks: semantic network attacks. Communications of the ACM, 43(12):168-168, December 2000.

$\left[\mathrm{SKK}^{+}\right.$97] Christoph L. Schuba, Ivan V. Krsul, Markus G. Kuhn, Eugene H. Spafford, Aurobindo Sundaram, and Diego Zamboni. Analysis of a denial of service attack on TCP. In Proceedings of the 1997 IEEE Symposium on Security and Privacy, pages 208-223. IEEE Computer Society, IEEE Computer Society Press, May 1997.

[SP01] Dawn X. Song and Adrian Perrig. Advanced and authenticated marking schemes for IP traceback. In Proceedings of IEEE Infocom 2001, April 2001.

[SWKA00] Stefan Savage, David Wetherall, Anna R. Karlin, and Tom Anderson. Practical network support for IP traceback. In Proceedings of the 2000 ACM SIGCOMM Conference, pages 295-306, August 2000.

[The03] The Honeynet Project. Know Your Enemy: GenII Honeynets, November 2003. http://www . honeynet .org/papers/gen2/.

[The05] The Honeynet Project. Know your Enemy: Tracking Botnets, March 2005. http: //www.honeynet.org/papers/bots. 


\section{Aachener Informatik-Berichte}

This is a list of recent technical reports. To obtain copies of technical reports please consult http://aib.informatik.rwth-aachen.de/ or send your request to: Informatik-Bibliothek, RWTH Aachen, Ahornstr. 55, 52056 Aachen, Email: biblio@informatik.rwth-aachen.de

1987-01 * Fachgruppe Informatik: Jahresbericht 1986

1987-02 * David de Frutos Escrig, Klaus Indermark: Equivalence Relations of NonDeterministic Ianov-Schemes

1987-03 * Manfred Nagl: A Software Development Environment based on Graph Technology

1987-04* Claus Lewerentz, Manfred Nagl, Bernhard Westfechtel: On Integration Mechanisms within a Graph-Based Software Development Environment

1987-05 * Reinhard Rinn: Über Eingabeanomalien bei verschiedenen Inferenzmodellen

1987-06 * Werner Damm, Gert Döhmen: Specifying Distributed Computer Architectures in $\mathrm{AADL}^{*}$

1987-07 * Gregor Engels, Claus Lewerentz, Wilhelm Schäfer: Graph Grammar Engineering: A Software Specification Method

1987-08 * Manfred Nagl: Set Theoretic Approaches to Graph Grammars

1987-09 * Claus Lewerentz, Andreas Schürr: Experiences with a Database System for Software Documents

1987-10 * Herbert Klaeren, Klaus Indermark: A New Implementation Technique for Recursive Function Definitions

1987-11 * Rita Loogen: Design of a Parallel Programmable Graph Reduction Machine with Distributed Memory

1987-12 J. Börstler, U. Möncke, R. Wilhelm: Table compression for tree automata

1988-01 * Gabriele Esser, Johannes Rückert, Frank Wagner: Gesellschaftliche Aspekte der Informatik

1988-02 * Peter Martini, Otto Spaniol: Token-Passing in High-Speed Backbone Networks for Campus-Wide Environments

1988-03 * Thomas Welzel: Simulation of a Multiple Token Ring Backbone

1988-04 * Peter Martini: Performance Comparison for HSLAN Media Access Protocols

1988-05 * Peter Martini: Performance Analysis of Multiple Token Rings

1988-06 * Andreas Mann, Johannes Rückert, Otto Spaniol: Datenfunknetze

1988-07 * Andreas Mann, Johannes Rückert: Packet Radio Networks for Data Exchange

1988-08 * Andreas Mann, Johannes Rückert: Concurrent Slot Assignment Protocol for Packet Radio Networks

1988-09 * W. Kremer, F. Reichert, J. Rückert, A. Mann: Entwurf einer Netzwerktopologie für ein Mobilfunknetz zur Unterstützung des öffentlichen Straßenverkehrs

1988-10 * Kai Jakobs: Towards User-Friendly Networking

1988-11 * Kai Jakobs: The Directory - Evolution of a Standard

1988-12 * Kai Jakobs: Directory Services in Distributed Systems - A Survey

1988-13 * Martine Schümmer: RS-511, a Protocol for the Plant Floor 
1988-14* U. Quernheim: Satellite Communication Protocols - A Performance Comparison Considering On-Board Processing

1988-15 * Peter Martini, Otto Spaniol, Thomas Welzel: File Transfer in High Speed Token Ring Networks: Performance Evaluation by Approximate Analysis and Simulation

1988-16 * Fachgruppe Informatik: Jahresbericht 1987

1988-17* Wolfgang Thomas: Automata on Infinite Objects

1988-18 * Michael Sonnenschein: On Petri Nets and Data Flow Graphs

1988-19* Heiko Vogler: Functional Distribution of the Contextual Analysis in Block-Structured Programming Languages: A Case Study of Tree Transducers

1988-20 * Thomas Welzel: Einsatz des Simulationswerkzeuges QNAP2 zur Leistungsbewertung von Kommunikationsprotokollen

1988-21 * Th. Janning, C. Lewerentz: Integrated Project Team Management in a Software Development Environment

1988-22 * Joost Engelfriet, Heiko Vogler: Modular Tree Transducers

1988-23 * Wolfgang Thomas: Automata and Quantifier Hierarchies

1988-24 * Uschi Heuter: Generalized Definite Tree Languages

1989-01 * Fachgruppe Informatik: Jahresbericht 1988

1989-02 * G. Esser, J. Rückert, F. Wagner (Hrsg.): Gesellschaftliche Aspekte der Informatik

1989-03 * Heiko Vogler: Bottom-Up Computation of Primitive Recursive Tree Functions

1989-04 * Andy Schürr: Introduction to PROGRESS, an Attribute Graph Grammar Based Specification Language

1989-05 J. Börstler: Reuse and Software Development - Problems, Solutions, and Bibliography (in German)

1989-06 * Kai Jakobs: OSI - An Appropriate Basis for Group Communication?

1989-07* Kai Jakobs: ISO's Directory Proposal - Evolution, Current Status and Future Problems

1989-08 * Bernhard Westfechtel: Extension of a Graph Storage for Software Documents with Primitives for Undo/Redo and Revision Control

1989-09 * Peter Martini: High Speed Local Area Networks - A Tutorial

1989-10 * P. Davids, Th. Welzel: Performance Analysis of DQDB Based on Simulation

1989-11 * Manfred Nagl (Ed.): Abstracts of Talks presented at the WG'89 15th International Workshop on Graphtheoretic Concepts in Computer Science

1989-12 * Peter Martini: The DQDB Protocol - Is it Playing the Game?

1989-13 * Martine Schümmer: CNC/DNC Communication with MAP

1989-14 * Martine Schümmer: Local Area Networks for Manufactoring Environments with hard Real-Time Requirements

1989-15* M. Schümmer, Th. Welzel, P. Martini: Integration of Field Bus and MAP Networks - Hierarchical Communication Systems in Production Environments

1989-16 * G. Vossen, K.-U. Witt: SUXESS: Towards a Sound Unification of Extensions of the Relational Data Model

1989-17* J. Derissen, P. Hruschka, M.v.d. Beeck, Th. Janning, M. Nagl: Integrating Structured Analysis and Information Modelling 
1989-18 A. Maassen: Programming with Higher Order Functions

1989-19 * Mario Rodriguez-Artalejo, Heiko Vogler: A Narrowing Machine for Syntax Directed BABEL

1989-20 H. Kuchen, R. Loogen, J.J. Moreno Navarro, M. Rodriguez Artalejo: Graph-based Implementation of a Functional Logic Language

1990-01 * Fachgruppe Informatik: Jahresbericht 1989

1990-02 * Vera Jansen, Andreas Potthoff, Wolfgang Thomas, Udo Wermuth: A Short Guide to the AMORE System (Computing Automata, MOnoids and Regular Expressions)

1990-03 * Jerzy Skurczynski: On Three Hierarchies of Weak SkS Formulas

1990-04 R. Loogen: Stack-based Implementation of Narrowing

1990-05 H. Kuchen, A. Wagener: Comparison of Dynamic Load Balancing Strategies

1990-06 * Kai Jakobs, Frank Reichert: Directory Services for Mobile Communication

1990-07* Kai Jakobs: What's Beyond the Interface - OSI Networks to Support Cooperative Work

1990-08 * Kai Jakobs: Directory Names and Schema - An Evaluation

1990-09 * Ulrich Quernheim, Dieter Kreuer: Das CCITT - Signalisierungssystem Nr. 7 auf Satellitenstrecken; Simulation der Zeichengabestrecke

1990-11 H. Kuchen, R. Loogen, J.J. Moreno Navarro, M. Rodriguez Artalejo: Lazy Narrowing in a Graph Machine

1990-12 * Kai Jakobs, Josef Kaltwasser, Frank Reichert, Otto Spaniol: Der Computer fährt mit

1990-13 * Rudolf Mathar, Andreas Mann: Analyzing a Distributed Slot Assignment Protocol by Markov Chains

1990-14 A. Maassen: Compilerentwicklung in Miranda - ein Praktikum in funktionaler Programmierung (written in german)

1990-15 * Manfred Nagl, Andreas Schürr: A Specification Environment for Graph Grammars

1990-16 A. Schürr: PROGRESS: A VHL-Language Based on Graph Grammars

1990-17 * Marita Möller: Ein Ebenenmodell wissensbasierter Konsultationen - Unterstützung für Wissensakquisition und Erklärungsfähigkeit

1990-18 * Eric Kowalewski: Entwurf und Interpretation einer Sprache zur Beschreibung von Konsultationsphasen in Expertensystemen

1990-20 Y. Ortega Mallen, D. de Frutos Escrig: A Complete Proof System for Timed Observations

1990-21 * Manfred Nagl: Modelling of Software Architectures: Importance, Notions, Experiences

1990-22 H. Fassbender, H. Vogler: A Call-by-need Implementation of Syntax Directed Functional Programming

1991-01 Guenther Geiler (ed.), Fachgruppe Informatik: Jahresbericht 1990

1991-03 B. Steffen, A. Ingolfsdottir: Characteristic Formulae for Processes with Divergence

1991-04 M. Portz: A new class of cryptosystems based on interconnection networks

1991-05 H. Kuchen, G. Geiler: Distributed Applicative Arrays

1991-06 * Ludwig Staiger: Kolmogorov Complexity and Hausdorff Dimension 
1991-07* Ludwig Staiger: Syntactic Congruences for w-languages

1991-09 * Eila Kuikka: A Proposal for a Syntax-Directed Text Processing System

1991-10 K. Gladitz, H. Fassbender, H. Vogler: Compiler-based Implementation of Syntax-Directed Functional Programming

1991-11 R. Loogen, St. Winkler: Dynamic Detection of Determinism in Functional Logic Languages

1991-12 * K. Indermark, M. Rodriguez Artalejo (Eds.): Granada Workshop on the Integration of Functional and Logic Programming

1991-13 * Rolf Hager, Wolfgang Kremer: The Adaptive Priority Scheduler: A More Fair Priority Service Discipline

1991-14 * Andreas Fasbender, Wolfgang Kremer: A New Approximation Algorithm for Tandem Networks with Priority Nodes

1991-15 J. Börstler, A. Zündorf: Revisiting extensions to Modula-2 to support reusability

1991-16 J. Börstler, Th. Janning: Bridging the gap between Requirements Analysis and Design

1991-17 A. Zündorf, A. Schürr: Nondeterministic Control Structures for Graph Rewriting Systems

1991-18 * Matthias Jarke, John Mylopoulos, Joachim W. Schmidt, Yannis Vassiliou: DAIDA: An Environment for Evolving Information Systems

1991-19 M. Jeusfeld, M. Jarke: From Relational to Object-Oriented Integrity Simplification

1991-20 G. Hogen, A. Kindler, R. Loogen: Automatic Parallelization of Lazy Functional Programs

1991-21 * Prof. Dr. rer. nat. Otto Spaniol: ODP (Open Distributed Processing): Yet another Viewpoint

1991-22 H. Kuchen, F. Lücking, H. Stoltze: The Topology Description Language TDL

1991-23 S. Graf, B. Steffen: Compositional Minimization of Finite State Systems

1991-24 R. Cleaveland, J. Parrow, B. Steffen: The Concurrency Workbench: A Semantics Based Tool for the Verification of Concurrent Systems

1991-25 * Rudolf Mathar, Jürgen Mattfeldt: Optimal Transmission Ranges for Mobile Communication in Linear Multihop Packet Radio Networks

1991-26 M. Jeusfeld, M. Staudt: Query Optimization in Deductive Object Bases

1991-27 J. Knoop, B. Steffen: The Interprocedural Coincidence Theorem

1991-28 J. Knoop, B. Steffen: Unifying Strength Reduction and Semantic Code Motion

1991-30 T. Margaria: First-Order theories for the verification of complex FSMs

1991-31 B. Steffen: Generating Data Flow Analysis Algorithms from Modal Specifications

1992-01 Stefan Eherer (ed.), Fachgruppe Informatik: Jahresbericht 1991

1992-02 * Bernhard Westfechtel: Basismechanismen zur Datenverwaltung in strukturbezogenen Hypertextsystemen

1992-04 S. A. Smolka, B. Steffen: Priority as Extremal Probability

1992-05* Matthias Jarke, Carlos Maltzahn, Thomas Rose: Sharing Processes: Team Coordination in Design Repositories

1992-06 O. Burkart, B. Steffen: Model Checking for Context-Free Processes 
1992-07* Matthias Jarke, Klaus Pohl: Information Systems Quality and Quality Information Systems

1992-08 * Rudolf Mathar, Jürgen Mattfeldt: Analyzing Routing Strategy NFP in Multihop Packet Radio Networks on a Line

1992-09 * Alfons Kemper, Guido Moerkotte: Grundlagen objektorientierter Datenbanksysteme

1992-10 Matthias Jarke, Manfred Jeusfeld, Andreas Miethsam, Michael Gocek: Towards a logic-based reconstruction of software configuration management

1992-11 Werner Hans: A Complete Indexing Scheme for WAM-based Abstract Machines

1992-12 W. Hans, R. Loogen, St. Winkler: On the Interaction of Lazy Evaluation and Backtracking

1992-13 * Matthias Jarke, Thomas Rose: Specification Management with CAD

1992-14 Th. Noll, H. Vogler: Top-down Parsing with Simultaneous Evaluation on Noncircular Attribute Grammars

1992-15 A. Schuerr, B. Westfechtel: Graphgrammatiken und Graphersetzungssysteme(written in german)

1992-16 * Graduiertenkolleg Informatik und Technik (Hrsg.): Forschungsprojekte des Graduiertenkollegs Informatik und Technik

1992-17 M. Jarke (ed.): ConceptBase V3.1 User Manual

1992-18 * Clarence A. Ellis, Matthias Jarke (Eds.): Distributed Cooperation in Integrated Information Systems - Proceedings of the Third International Workshop on Intelligent and Cooperative Information Systems

1992-19-00 H. Kuchen, R. Loogen (eds.): Proceedings of the 4th Int. Workshop on the Parallel Implementation of Functional Languages

1992-19-01 G. Hogen, R. Loogen: PASTEL - A Parallel Stack-Based Implementation of Eager Functional Programs with Lazy Data Structures (Extended Abstract)

1992-19-02 H. Kuchen, K. Gladitz: Implementing Bags on a Shared Memory MIMDMachine

1992-19-03 C. Rathsack, S.B. Scholz: LISA - A Lazy Interpreter for a Full-Fledged Lambda-Calculus

1992-19-04 T.A. Bratvold: Determining Useful Parallelism in Higher Order Functions

1992-19-05 S. Kahrs: Polymorphic Type Checking by Interpretation of Code

1992-19-06 M. Chakravarty, M. Köhler: Equational Constraints, Residuation, and the Parallel JUMP-Machine

1992-19-07 J. Seward: Polymorphic Strictness Analysis using Frontiers (Draft Version)

1992-19-08 D. Gärtner, A. Kimms, W. Kluge: pi-Red^+ - A Compiling GraphReduction System for a Full Fledged Lambda-Calculus

1992-19-09 D. Howe, G. Burn: Experiments with strict STG code

1992-19-10 J. Glauert: Parallel Implementation of Functional Languages Using Small Processes

1992-19-11 M. Joy, T. Axford: A Parallel Graph Reduction Machine

1992-19-12 A. Bennett, P. Kelly: Simulation of Multicache Parallel Reduction 
1992-19-13 K. Langendoen, D.J. Agterkamp: Cache Behaviour of Lazy Functional Programs (Working Paper)

1992-19-14 K. Hammond, S. Peyton Jones: Profiling scheduling strategies on the GRIP parallel reducer

1992-19-15 S. Mintchev: Using Strictness Information in the STG-machine

1992-19-16 D. Rushall: An Attribute Grammar Evaluator in Haskell

1992-19-17 J. Wild, H. Glaser, P. Hartel: Statistics on storage management in a lazy functional language implementation

1992-19-18 W.S. Martins: Parallel Implementations of Functional Languages

1992-19-19 D. Lester: Distributed Garbage Collection of Cyclic Structures (Draft version)

1992-19-20 J.C. Glas, R.F.H. Hofman, W.G. Vree: Parallelization of Branch-andBound Algorithms in a Functional Programming Environment

1992-19-21 S. Hwang, D. Rushall: The nu-STG machine: a parallelized Spineless Tagless Graph Reduction Machine in a distributed memory architecture (Draft version)

1992-19-22 G. Burn, D. Le Metayer: Cps-Translation and the Correctness of Optimising Compilers

1992-19-23 S.L. Peyton Jones, P. Wadler: Imperative functional programming (Brief summary)

1992-19-24 W. Damm, F. Liu, Th. Peikenkamp: Evaluation and Parallelization of Functions in Functional + Logic Languages (abstract)

1992-19-25 M. Kesseler: Communication Issues Regarding Parallel Functional Graph Rewriting

1992-19-26 Th. Peikenkamp: Charakterizing and representing neededness in functional loginc languages (abstract)

1992-19-27 H. Doerr: Monitoring with Graph-Grammars as formal operational Models

1992-19-28 J. van Groningen: Some implementation aspects of Concurrent Clean on distributed memory architectures

1992-19-29 G. Ostheimer: Load Bounding for Implicit Parallelism (abstract)

1992-20 H. Kuchen, F.J. Lopez Fraguas, J.J. Moreno Navarro, M. Rodriguez Artalejo: Implementing Disequality in a Lazy Functional Logic Language

1992-21 H. Kuchen, F.J. Lopez Fraguas: Result Directed Computing in a Functional Logic Language

1992-22 H. Kuchen, J.J. Moreno Navarro, M.V. Hermenegildo: Independent AND-Parallel Narrowing

1992-23 T. Margaria, B. Steffen: Distinguishing Formulas for Free

1992-24 K. Pohl: The Three Dimensions of Requirements Engineering

1992-25 * R. Stainov: A Dynamic Configuration Facility for Multimedia Communications

1992-26 * Michael von der Beeck: Integration of Structured Analysis and Timed Statecharts for Real-Time and Concurrency Specification

1992-27 W. Hans, St. Winkler: Aliasing and Groundness Analysis of Logic Programs through Abstract Interpretation and its Safety

1992-28 * Gerhard Steinke, Matthias Jarke: Support for Security Modeling in Information Systems Design

1992-29 B. Schinzel: Warum Frauenforschung in Naturwissenschaft und Technik 
1992-30 A. Kemper, G. Moerkotte, K. Peithner: Object-Orientation Axiomatised by Dynamic Logic

1992-32 * Bernd Heinrichs, Kai Jakobs: Timer Handling in High-Performance Transport Systems

1992-33 * B. Heinrichs, K. Jakobs, K. Lenßen, W. Reinhardt, A. Spinner: EuroBridge: Communication Services for Multimedia Applications

1992-34 C. Gerlhof, A. Kemper, Ch. Kilger, G. Moerkotte: Partition-Based Clustering in Object Bases: From Theory to Practice

1992-35 J. Börstler: Feature-Oriented Classification and Reuse in IPSEN

1992-36 M. Jarke, J. Bubenko, C. Rolland, A. Sutcliffe, Y. Vassiliou: Theories Underlying Requirements Engineering: An Overview of NATURE at Genesis

1992-37 * K. Pohl, M. Jarke: Quality Information Systems: Repository Support for Evolving Process Models

1992-38 A. Zuendorf: Implementation of the imperative / rule based language PROGRES

1992-39 P. Koch: Intelligentes Backtracking bei der Auswertung funktionallogischer Programme

1992-40 * Rudolf Mathar, Jürgen Mattfeldt: Channel Assignment in Cellular Radio Networks

1992-41 * Gerhard Friedrich, Wolfgang Neidl: Constructive Utility in Model-Based Diagnosis Repair Systems

1992-42 * P. S. Chen, R. Hennicker, M. Jarke: On the Retrieval of Reusable Software Components

1992-43 W. Hans, St.Winkler: Abstract Interpretation of Functional Logic Languages

1992-44 N. Kiesel, A. Schuerr, B. Westfechtel: Design and Evaluation of GRAS, a Graph-Oriented Database System for Engineering Applications

1993-01 * Fachgruppe Informatik: Jahresbericht 1992

1993-02 * Patrick Shicheng Chen: On Inference Rules of Logic-Based Information Retrieval Systems

1993-03 G. Hogen, R. Loogen: A New Stack Technique for the Management of Runtime Structures in Distributed Environments

1993-05 A. Zündorf: A Heuristic for the Subgraph Isomorphism Problem in Executing PROGRES

1993-06 A. Kemper, D. Kossmann: Adaptable Pointer Swizzling Strategies in Object Bases: Design, Realization, and Quantitative Analysis

1993-07 * Graduiertenkolleg Informatik und Technik (Hrsg.): Graduiertenkolleg Informatik und Technik

1993-08 * Matthias Berger: k-Coloring Vertices using a Neural Network with Convergence to Valid Solutions

1993-09 M. Buchheit, M. Jeusfeld, W. Nutt, M. Staudt: Subsumption between Queries to Object-Oriented Databases

1993-10 O. Burkart, B. Steffen: Pushdown Processes: Parallel Composition and Model Checking

1993-11 * R. Große-Wienker, O. Hermanns, D. Menzenbach, A. Pollacks, S. Repetzki, J. Schwartz, K. Sonnenschein, B. Westfechtel: Das SUKITSProjekt: A-posteriori-Integration heterogener CIM-Anwendungssysteme 
1993-12 * Rudolf Mathar, Jürgen Mattfeldt: On the Distribution of Cumulated Interference Power in Rayleigh Fading Channels

1993-13 O. Maler, L. Staiger: On Syntactic Congruences for omega-languages

1993-14 M. Jarke, St. Eherer, R. Gallersdoerfer, M. Jeusfeld, M. Staudt: ConceptBase - A Deductive Object Base Manager

1993-15 M. Staudt, H.W. Nissen, M.A. Jeusfeld: Query by Class, Rule and Concept

1993-16 * M. Jarke, K. Pohl, St. Jacobs et al.: Requirements Engineering: An Integrated View of Representation Process and Domain

1993-17* M. Jarke, K. Pohl: Establishing Vision in Context: Towards a Model of Requirements Processes

1993-18 W. Hans, H. Kuchen, St. Winkler: Full Indexing for Lazy Narrowing

1993-19 W. Hans, J.J. Ruz, F. Saenz, St. Winkler: A VHDL Specification of a Shared Memory Parallel Machine for Babel

1993-20* K. Finke, M. Jarke, P. Szczurko, R. Soltysiak: Quality Management for Expert Systems in Process Control

1993-21 M. Jarke, M.A. Jeusfeld, P. Szczurko: Three Aspects of Intelligent Cooperation in the Quality Cycle

1994-01 Margit Generet, Sven Martin (eds.), Fachgruppe Informatik: Jahresbericht 1993

1994-02 M. Lefering: Development of Incremental Integration Tools Using Formal Specifications

1994-03 * P. Constantopoulos, M. Jarke, J. Mylopoulos, Y. Vassiliou: The Software Information Base: A Server for Reuse

1994-04 * Rolf Hager, Rudolf Mathar, Jürgen Mattfeldt: Intelligent Cruise Control and Reliable Communication of Mobile Stations

1994-05 * Rolf Hager, Peter Hermesmann, Michael Portz: Feasibility of Authentication Procedures within Advanced Transport Telematics

1994-06 * Claudia Popien, Bernd Meyer, Axel Kuepper: A Formal Approach to Service Import in ODP Trader Federations

1994-07 P. Peters, P. Szczurko: Integrating Models of Quality Management Methods by an Object-Oriented Repository

1994-08 * Manfred Nagl, Bernhard Westfechtel: A Universal Component for the Administration in Distributed and Integrated Development Environments

1994-09 * Patrick Horster, Holger Petersen: Signatur- und Authentifikationsverfahren auf der Basis des diskreten Logarithmusproblems

1994-11 A. Schürr: PROGRES, A Visual Language and Environment for PROgramming with Graph REwrite Systems

1994-12 A. Schürr: Specification of Graph Translators with Triple Graph Grammars

1994-13 A. Schürr: Logic Based Programmed Structure Rewriting Systems

1994-14 L. Staiger: Codes, Simplifying Words, and Open Set Condition

1994-15 * Bernhard Westfechtel: A Graph-Based System for Managing Configurations of Engineering Design Documents

1994-16 P. Klein: Designing Software with Modula-3

1994-17 I. Litovsky, L. Staiger: Finite acceptance of infinite words 
1994-18 G. Hogen, R. Loogen: Parallel Functional Implementations: Graphbased vs. Stackbased Reduction

1994-19 M. Jeusfeld, U. Johnen: An Executable Meta Model for Re-Engineering of Database Schemas

1994-20 * R. Gallersdörfer, M. Jarke, K. Klabunde: Intelligent Networks as a Data Intensive Application (INDIA)

1994-21 M. Mohnen: Proving the Correctness of the Static Link Technique Using Evolving Algebras

1994-22 H. Fernau, L. Staiger: Valuations and Unambiguity of Languages, with Applications to Fractal Geometry

1994-24* M. Jarke, K. Pohl, R. Dömges, St. Jacobs, H. W. Nissen: Requirements Information Management: The NATURE Approach

1994-25 * M. Jarke, K. Pohl, C. Rolland, J.-R. Schmitt: Experience-Based Method Evaluation and Improvement: A Process Modeling Approach

1994-26 * St. Jacobs, St. Kethers: Improving Communication and Decision Making within Quality Function Deployment

1994-27* M. Jarke, H. W. Nissen, K. Pohl: Tool Integration in Evolving Information Systems Environments

1994-28 O. Burkart, D. Caucal, B. Steffen: An Elementary Bisimulation Decision Procedure for Arbitrary Context-Free Processes

1995-01 * Fachgruppe Informatik: Jahresbericht 1994

1995-02 Andy Schürr, Andreas J. Winter, Albert Zündorf: Graph Grammar Engineering with PROGRES

1995-03 Ludwig Staiger: A Tight Upper Bound on Kolmogorov Complexity by Hausdorff Dimension and Uniformly Optimal Prediction

1995-04 Birgitta König-Ries, Sven Helmer, Guido Moerkotte: An experimental study on the complexity of left-deep join ordering problems for cyclic queries

1995-05 Sophie Cluet, Guido Moerkotte: Efficient Evaluation of Aggregates on Bulk Types

1995-06 Sophie Cluet, Guido Moerkotte: Nested Queries in Object Bases

1995-07 Sophie Cluet, Guido Moerkotte: Query Optimization Techniques Exploiting Class Hierarchies

1995-08 Markus Mohnen: Efficient Compile-Time Garbage Collection for Arbitrary Data Structures

1995-09 Markus Mohnen: Functional Specification of Imperative Programs: An Alternative Point of View of Functional Languages

1995-10 Rainer Gallersdörfer, Matthias Nicola: Improving Performance in Replicated Databases through Relaxed Coherency

1995-11 * M.Staudt, K.von Thadden: Subsumption Checking in Knowledge Bases

1995-12 * G.V.Zemanek, H.W.Nissen, H.Hubert, M.Jarke: Requirements Analysis from Multiple Perspectives: Experiences with Conceptual Modeling Technology

1995-13 * M.Staudt, M.Jarke: Incremental Maintenance of Externally Materialized Views

1995-14 * P.Peters, P.Szczurko, M.Jeusfeld: Oriented Information Management: Conceptual Models at Work 
1995-15* Matthias Jarke, Sudha Ram (Hrsg.): WITS 95 Proceedings of the 5th Annual Workshop on Information Technologies and Systems

1995-16 * W.Hans, St.Winkler, F.Saenz: Distributed Execution in Functional Logic Programming

1996-01 * Jahresbericht 1995

1996-02 Michael Hanus, Christian Prehofer: Higher-Order Narrowing with Definitional Trees

1996-03 * W.Scheufele, G.Moerkotte: Optimal Ordering of Selections and Joins in Acyclic Queries with Expensive Predicates

1996-04 Klaus Pohl: PRO-ART: Enabling Requirements Pre-Traceability

1996-05 Klaus Pohl: Requirements Engineering: An Overview

1996-06 * M.Jarke, W.Marquardt: Design and Evaluation of Computer-Aided Process Modelling Tools

1996-07 Olaf Chitil: The Sigma-Semantics: A Comprehensive Semantics for Functional Programs

1996-08 * S.Sripada: On Entropy and the Limitations of the Second Law of Thermodynamics

1996-09 Michael Hanus (Ed.): Proceedings of the Poster Session of ALP96 - Fifth International Conference on Algebraic and Logic Programming

1996-09-0 Michael Hanus (Ed.): Proceedings of the Poster Session of ALP 96 Fifth International Conference on Algebraic and Logic Programming: Introduction and table of contents

1996-09-1 Ilies Alouini: An Implementation of Conditional Concurrent Rewriting on Distributed Memory Machines

1996-09-2 Olivier Danvy, Karoline Malmkjær: On the Idempotence of the CPS Transformation

1996-09-3 Víctor M. Gulías, José L. Freire: Concurrent Programming in Haskell

1996-09-4 Sébastien Limet, Pierre Réty: On Decidability of Unifiability Modulo Rewrite Systems

1996-09-5 Alexandre Tessier: Declarative Debugging in Constraint Logic Programming

1996-10 Reidar Conradi, Bernhard Westfechtel: Version Models for Software Configuration Management

1996-11 * C.Weise, D.Lenzkes: A Fast Decision Algorithm for Timed Refinement

1996-12 * R.Dömges, K.Pohl, M.Jarke, B.Lohmann, W.Marquardt: PROART/CE* — An Environment for Managing the Evolution of Chemical Process Simulation Models

1996-13 * K.Pohl, R.Klamma, K.Weidenhaupt, R.Dömges, P.Haumer, M.Jarke: A Framework for Process-Integrated Tools

1996-14 * R.Gallersdörfer, K.Klabunde, A.Stolz, M.Eßmajor: INDIA — Intelligent Networks as a Data Intensive Application, Final Project Report, June 1996

1996-15 * H.Schimpe, M.Staudt: VAREX: An Environment for Validating and Refining Rule Bases

1996-16 * M.Jarke, M.Gebhardt, S.Jacobs, H.Nissen: Conflict Analysis Across Heterogeneous Viewpoints: Formalization and Visualization

1996-17 Manfred A. Jeusfeld, Tung X. Bui: Decision Support Components on the Internet 
1996-18 Manfred A. Jeusfeld, Mike Papazoglou: Information Brokering: Design, Search and Transformation

1996-19 * P.Peters, M.Jarke: Simulating the impact of information flows in networked organizations

1996-20 Matthias Jarke, Peter Peters, Manfred A. Jeusfeld: Model-driven planning and design of cooperative information systems

1996-21 * G.de Michelis, E.Dubois, M.Jarke, F.Matthes, J.Mylopoulos, K.Pohl, J.Schmidt, C.Woo, E.Yu: Cooperative information systems: a manifesto

1996-22 * S.Jacobs, M.Gebhardt, S.Kethers, W.Rzasa: Filling HTML forms simultaneously: CoWeb architecture and functionality

1996-23 * M.Gebhardt, S.Jacobs: Conflict Management in Design

1997-01 Michael Hanus, Frank Zartmann (eds.): Jahresbericht 1996

1997-02 Johannes Faassen: Using full parallel Boltzmann Machines for Optimization

1997-03 Andreas Winter, Andy Schürr: Modules and Updatable Graph Views for PROgrammed Graph REwriting Systems

1997-04 Markus Mohnen, Stefan Tobies: Implementing Context Patterns in the Glasgow Haskell Compiler

1997-05 * S.Gruner: Schemakorrespondenzaxiome unterstützen die paargrammatische Spezifikation inkrementeller Integrationswerkzeuge

1997-06 Matthias Nicola, Matthias Jarke: Design and Evaluation of Wireless Health Care Information Systems in Developing Countries

1997-07 Petra Hofstedt: Taskparallele Skelette für irregulär strukturierte Probleme in deklarativen Sprachen

1997-08 Dorothea Blostein, Andy Schürr: Computing with Graphs and Graph Rewriting

1997-09 Carl-Arndt Krapp, Bernhard Westfechtel: Feedback Handling in Dynamic Task Nets

1997-10 Matthias Nicola, Matthias Jarke: Integrating Replication and Communication in Performance Models of Distributed Databases

1997-11 * R. Klamma, P. Peters, M. Jarke: Workflow Support for Failure Management in Federated Organizations

1997-13 Markus Mohnen: Optimising the Memory Management of Higher-Order Functional Programs

1997-14 Roland Baumann: Client/Server Distribution in a Structure-Oriented Database Management System

1997-15 George Botorog: High-Level Parallel Programming and the Efficient Implementation of Numerical Algorithms

1998-01 * Fachgruppe Informatik: Jahresbericht 1997

1998-02 Stefan Gruner, Manfred Nagel, Andy Schürr: Fine-grained and Structure-Oriented Document Integration Tools are Needed for Development Processes

1998-03 Stefan Gruner: Einige Anmerkungen zur graphgrammatischen Spezifikation von Integrationswerkzeugen nach Westfechtel, Janning, Lefering und Schürr

1998-04 * O. Kubitz: Mobile Robots in Dynamic Environments 
1998-05 Martin Leucker, Stephan Tobies: Truth - A Verification Platform for Distributed Systems

1998-06 * Matthias Oliver Berger: DECT in the Factory of the Future

1998-07 M. Arnold, M. Erdmann, M. Glinz, P. Haumer, R. Knoll, B. Paech, K. Pohl, J. Ryser, R. Studer, K. Weidenhaupt: Survey on the Scenario Use in Twelve Selected Industrial Projects

1998-08 * H. Aust: Sprachverstehen und Dialogmodellierung in natürlichsprachlichen Informationssystemen

1998-09 * Th. Lehmann: Geometrische Ausrichtung medizinischer Bilder am Beispiel intraoraler Radiographien

1998-10 * M. Nicola, M. Jarke: Performance Modeling of Distributed and Replicated Databases

1998-11 * Ansgar Schleicher, Bernhard Westfechtel, Dirk Jäger: Modeling Dynamic Software Processes in UML

1998-12 * W. Appelt, M. Jarke: Interoperable Tools for Cooperation Support using the World Wide Web

1998-13 Klaus Indermark: Semantik rekursiver Funktionsdefinitionen mit Striktheitsinformation

1999-01 * Jahresbericht 1998

1999-02 * F. Huch: Verifcation of Erlang Programs using Abstract Interpretation and Model Checking — Extended Version

1999-03 * R. Gallersdörfer, M. Jarke, M. Nicola: The ADR Replication Manager

1999-04 María Alpuente, Michael Hanus, Salvador Lucas, Germán Vidal: Specialization of Functional Logic Programs Based on Needed Narrowing

1999-05 * W. Thomas (Ed.): DLT 99 - Developments in Language Theory Fourth International Conference

1999-06 * Kai Jakobs, Klaus-Dieter Kleefeld: Informationssysteme für die angewandte historische Geographie

1999-07 Thomas Wilke: CTL+ is exponentially more succinct than CTL

1999-08 Oliver Matz: Dot-Depth and Monadic Quantifier Alternation over Pictures

2000-01 * Jahresbericht 1999

2000-02 Jens Vöge, Marcin Jurdzinski: A Discrete Strategy Improvement Algorithm for Solving Parity Games

2000-04 Andreas Becks, Stefan Sklorz, Matthias Jarke: Exploring the Semantic Structure of Technical Document Collections: A Cooperative Systems Approach

2000-05 Mareike Schoop: Cooperative Document Management

2000-06 Mareike Schoop, Christoph Quix (eds.): Proceedings of the Fifth International Workshop on the Language-Action Perspective on Communication Modelling

2000-07* Markus Mohnen, Pieter Koopman (Eds.): Proceedings of the 12th International Workshop of Functional Languages

2000-08 Thomas Arts, Thomas Noll: Verifying Generic Erlang Client-Server Implementations

2001-01 * Jahresbericht 2000

2001-02 Benedikt Bollig, Martin Leucker: Deciding LTL over Mazurkiewicz Traces 
2001-03 Thierry Cachat: The power of one-letter rational languages

2001-04 Benedikt Bollig, Martin Leucker, Michael Weber: Local Parallel Model Checking for the Alternation Free mu-Calculus

2001-05 Benedikt Bollig, Martin Leucker, Thomas Noll: Regular MSC Languages

2001-06 Achim Blumensath: Prefix-Recognisable Graphs and Monadic SecondOrder Logic

2001-07 Martin Grohe, Stefan Wöhrle: An Existential Locality Theorem

2001-08 Mareike Schoop, James Taylor (eds.): Proceedings of the Sixth International Workshop on the Language-Action Perspective on Communication Modelling

2001-09 Thomas Arts, Jürgen Giesl: A collection of examples for termination of term rewriting using dependency pairs

2001-10 Achim Blumensath: Axiomatising Tree-interpretable Structures

2001-11 Klaus Indermark, Thomas Noll (eds.): Kolloquium Programmiersprachen und Grundlagen der Programmierung

2002-01 * Jahresbericht 2001

2002-02 Jürgen Giesl, Aart Middeldorp: Transformation Techniques for ContextSensitive Rewrite Systems

2002-03 Benedikt Bollig, Martin Leucker, Thomas Noll: Generalised Regular MSC Languages

2002-04 Jürgen Giesl, Aart Middeldorp: Innermost Termination of ContextSensitive Rewriting

2002-05 Horst Lichter, Thomas von der Maßen, Thomas Weiler: Modelling Requirements and Architectures for Software Product Lines

2002-06 Henry N. Adorna: 3-Party Message Complexity is Better than 2-Party Ones for Proving Lower Bounds on the Size of Minimal Nondeterministic Finite Automata

2002-07 Jörg Dahmen: Invariant Image Object Recognition using Gaussian Mixture Densities

2002-08 Markus Mohnen: An Open Framework for Data-Flow Analysis in Java 2002-09 Markus Mohnen: Interfaces with Default Implementations in Java

2002-10 Martin Leucker: Logics for Mazurkiewicz traces

2002-11 Jürgen Giesl, Hans Zantema: Liveness in Rewriting

2003-01 * Jahresbericht 2002

2003-02 Jürgen Giesl, René Thiemann: Size-Change Termination for Term Rewriting

2003-03 Jürgen Giesl, Deepak Kapur: Deciding Inductive Validity of Equations

2003-04 Jürgen Giesl, René Thiemann, Peter Schneider-Kamp, Stephan Falke: Improving Dependency Pairs

2003-05 Christof Löding, Philipp Rohde: Solving the Sabotage Game is PSPACEhard

2003-06 Franz Josef Och: Statistical Machine Translation: From Single-Word Models to Alignment Templates

2003-07 Horst Lichter, Thomas von der Maßen, Alexander Nyßen, Thomas Weiler: Vergleich von Ansätzen zur Feature Modellierung bei der Softwareproduktlinienentwicklung

2003-08 Jürgen Giesl, René Thiemann, Peter Schneider-Kamp, Stephan Falke: Mechanizing Dependency Pairs 
2004-01 * Fachgruppe Informatik: Jahresbericht 2003

2004-02 Benedikt Bollig, Martin Leucker: Message-Passing Automata are expressively equivalent to EMSO logic

2004-03 Delia Kesner, Femke van Raamsdonk, Joe Wells (eds.): HOR 2004 - 2nd International Workshop on Higher-Order Rewriting

2004-04 Slim Abdennadher, Christophe Ringeissen (eds.): RULE 04 - Fifth International Workshop on Rule-Based Programming

2004-05 Herbert Kuchen (ed.): WFLP 04 - 13th International Workshop on Functional and (Constraint) Logic Programming

2004-06 Sergio Antoy, Yoshihito Toyama (eds.): WRS 04 - 4th International Workshop on Reduction Strategies in Rewriting and Programming

2004-07 Michael Codish, Aart Middeldorp (eds.): WST 04 - 7th International Workshop on Termination

2004-08 Klaus Indermark, Thomas Noll: Algebraic Correctness Proofs for Compiling Recursive Function Definitions with Strictness Information

2004-09 Joachim Kneis, Daniel Mölle, Stefan Richter, Peter Rossmanith: Parameterized Power Domination Complexity

2004-10 Zinaida Benenson, Felix C. Gärtner, Dogan Kesdogan: Secure MultiParty Computation with Security Modules

2005-01 * Fachgruppe Informatik: Jahresbericht 2004

2005-02 Maximillian Dornseif, Felix C. Gärtner, Thorsten Holz, Martin Mink: An Offensive Approach to Teaching Information Security: „Aachen Summer School Applied IT Security“

2005-03 Jürgen Giesl, René Thiemann, Peter Schneider-Kamp: Proving and Disproving Termination of Higher-Order Functions

2005-04 Daniel Mölle, Stefan Richter, Peter Rossmanith: A Faster Algorithm for the Steiner Tree Problem

2005-05 Fabien Pouget, Thorsten Holz: A Pointillist Approach for Comparing Honeypots

2005-06 Simon Fischer, Berthold Vöcking: Adaptive Routing with Stale Information

* These reports are only available as a printed version.

Please contact biblio@informatik.rwth-aachen.de to obtain copies. 